\title{
Influência do Perfil Lipídico em Placas Ateroscleróticas Coronarianas Moderadas: Análise com Ultrassom Intracoronariano
}

\author{
Eduardo Missel', Gilson A. Yared², Francisco Costa1', Felipe Yared², Guilherme Yared², \\ Patrick Garcia de Lima ${ }^{2}$, Guilherme Lopes de Lima², Adroaldo Yared ${ }^{2}$, Marcos Sardinha', \\ Arnaldo Stier ${ }^{1}$, José Antonio da Silva ${ }^{1}$, Newton Stadler de Souza ${ }^{1}$
}

\section{RESUMO}

Introdução: O controle do perfil lipídico é uma das medidaschave para se reduzir a incidência de eventos cardíacos em coronariopatas. Objetivamos com este estudo determinar qual componente lipídico sérico tem maior influência no tamanho do ateroma por meio da análise de lesões coronarianas moderadas ao ultrassom intracoronariano. Método: Realizamos análise com ultrassom intracoronariano de 35 lesões moderadas em uma série consecutiva de 31 pacientes com indicação para o procedimento, realizando medidas das áreas da secção transversa (CSA) da membrana elástica externa (MEE), do lúmen, e da placa e média ( $P \& M)$, e a carga de placa na área luminal mínima lesional (ALM). Resultados: Os pacientes apresentavam média de idade de $64 \pm 11$ anos, sendo $75 \%$ homens e $22 \%$ diabéticos. Observamos associação inversa entre os níveis de colesterol de lipoproteína de alta densidade (HDLcolesterol) e área da $\mathrm{P} \& \mathrm{M}(\mathrm{r}=-0,40, \mathrm{P}=0,02)$. Colesterol total, colesterol de lipoproteína de baixa densidade (LDLcolesterol) ou triglicerídeos não apresentaram associação significativa com nenhuma medida do ateroma. A regressão multivariada em etapas revelou que, entre todos os fatores de risco cardiovascular, função ventricular, perfil lipídico e medicações anti-isquêmicas, somente os níveis de HDL-colesterol (coeficiente $=-0,58 ; \mathrm{P}=0,001$ ) e a fração de ejeção do ventrículo esquerdo (coeficiente $=-0,40$; $\mathrm{P}=0,02)$ foram preditores independentes da carga de placa. Pacientes com HDL-colesterol $>50 \mathrm{mg} / \mathrm{dl}$ apresentaram menor área da $\mathrm{P} \& M\left(4,9 \pm 3,5 \mathrm{~mm}^{2}\right.$ vs. $\left.8,84 \pm 3,4 \mathrm{~mm}^{2} ; \mathrm{P}=0,03\right)$ e da $\operatorname{MEE}\left(9,4 \pm 3,2 \mathrm{~mm}^{2}\right.$ vs. $\left.13,9 \pm 3,7 \mathrm{~mm}^{2} ; \mathrm{P}=0,01\right)$ quando comparados a pacientes com HDL-colesterol $\leq 50 \mathrm{mg} / \mathrm{dl}$. Conclusão: Neste estudo, o nível sérico de

\section{ABSTRACT}

Relationship Between Lipid Profile And Coronary Plaque Burden: An Intravascular Ultrasound Analysis

Objectives: Lipid profile control is a key measure in order to reduce the incidence of future cardiac events in the setting of coronary artery disease. We aim to determine which lipid profile parameter has greater influence on atheroma size using intravascular ultrasound analysis of moderate lesions. Methods: We performed intravascular ultrasound analysis in a consecutive series of 31 patients presenting 35 moderate lesions, measuring the cross-sectional area (CSA) of vessel, lumen, plaque \& media (P\&M) and plaque burden at the minimal lumen area. Results: Mean age was $64 \pm 11$ years, with $75 \%$ men and $22 \%$ diabetics. An inverse relationship was observed between high-density lipoprotein cholesterol (HDL-cholesterol) levels and P\&M area $(r=-0.40, P=0.02)$. Total cholesterol, low-density lipoprotein cholesterol (LDL-cholesterol) or triglycerides were not related to atheroma size. Multivariate regression revealed that among cardiovascular risk factors, ejection fraction, lipid profile and anti-ischemic drugs, only HDLcholesterol (Coefficient $=-0.58 ; \mathrm{P}=0.001)$ and left ventricular ejection fraction (Coefficient $=-0.40 ; \mathrm{P}=0.02$ ) were independent predictors of plaque burden. Patients with $\mathrm{HDL}$-cholesterol $>50 \mathrm{mg} / \mathrm{dl}$ presented smaller P\&M area $\left(4.9 \pm 3.5 \mathrm{~mm}^{2}\right.$ vs. $\left.8.84 \pm 3.4 \mathrm{~mm}^{2} ; \mathrm{P}=0.03\right)$, and vessel area $\left(9.4 \pm 3.2 \mathrm{~mm}^{2}\right.$ vs. $\left.13.9 \pm 3.7 \mathrm{~mm}^{2} ; \mathrm{P}=0.01\right)$ when compared to patients with HDL-cholesterol $\leq 50 \mathrm{mg} / \mathrm{dL}$. Conclusions: In this study, serum HDL-cholesterol level was the single independent predictor of coronary plaque

\footnotetext{
1 Instituto de Neurologia e Cardiologia de Curitiba (INC) - Curitiba, PR, Brasil.

2 Clínica Fisicor - Curitiba, PR, Brasil.

Correspondência: Eduardo Missel. Rua Jeremias Maciel Perretto, 300 - Ecoville - Curitiba, PR, Brasil - CEP 81210-310

E-mail: emissel@crf.org

Recebido em: 9/12/2008 • Aceito em: 16/3/2009
} 
Missel E, et al. Influência do Perfil Lipídico em Placas Ateroscleróticas Coronarianas Moderadas: Análise com Ultrassom Intracoronariano. Rev Bras Cardiol Invas. 2009;17(1):88-93.

HDL-colesterol foi o único componente do perfil lipídico capaz de predizer o tamanho do ateroma na ALM em lesões angiograficamente moderadas avaliadas ao ultrassom intracoronariano.

DESCRITORES: Lipídeos. Doença das coronárias. Arteriosclerose/ultrassonografia. Ultrassonografia de intervenção.

A s dislipidemias estão entre os fatores de risco cardiovascular que mais se correlacionam com eventos coronarianos futuros ${ }^{1-6}$. A hipercolesterolemia, em particular, tem influência prognóstica significativa na mortalidade coronariana, e níveis de lipoproteína de baixa densidade (LDL) inferiores a $70 \mathrm{mg} / \mathrm{dl}$ têm sido recomendados com o objetivo de reduzir a incidência de infarto agudo do miocárdio em pacientes suscetíveis, como medida de prevenção secundária. Entretanto, apesar desse efeito prognóstico inegável, a redução agressiva dos níveis de LDL acarreta apenas modesta redução do ateroma em estudos com ultrassom intracoronariano seriado $^{7-12}$.

O objetivo deste estudo é determinar a relação entre os componentes do perfil lipídico e o tamanho do ateroma quando acessado pela análise de lesões angiograficamente moderadas ao ultrassom intracoronariano, em uma tentativa de se definir qual fração lipídica condiciona em maior grau o acúmulo de placa aterosclerótica.

\section{MÉTODO}

\section{Protocolo do estudo e definições}

Entre agosto de 2007 e novembro de 2008, 31 pacientes com indicação de análise de lesão angiograficamente moderada ao ultrassom intracoronariano em nossa instituição foram incluídos neste registro prospectivo. Foram incluídas 35 lesões coronarianas de novo, nativas, únicas ou múltiplas (diâmetro de estenose de 40-60\% por estimativa visual à angiografia) em um ou mais vasos, que pudessem ser cruzadas com um cateter de ultrassom intracoronariano no momento do cateterismo ou pré-angioplastia e as medidas intracoronarianas realizadas na área luminal mínima (ALM).

Os comitês de ética das instituições participantes aprovaram o protocolo e o consentimento informado foi obtido dos pacientes participantes.

Dislipidemia foi definida como colesterol total $\geq 200 \mathrm{mg} / \mathrm{dl}$, LDL-colesterol $\geq 100 \mathrm{mg} / \mathrm{dl}$, colesterol de lipoproteína de alta densidade (HDL-colesterol) $<50 \mathrm{mg} / \mathrm{dl}$, triglicerídeos $\geq 150 \mathrm{mg} / \mathrm{dl}$ ou uso de terapia antilipemiante. Tabagismo foi definido como hábito de fumar atual ou interrompido há menos de um mês antes da inclusão no estudo. Hipertensão foi definida como pressão arterial sistólica $\geq 140 \mathrm{mmHg}$, burden at minimal lumen area of moderate lesions evaluated by intravascular ultrasound.

DESCRIPTORS: Lipids. Coronary disease. Arteriosclerosis/ ultrasonography. Ultrasonography, interventional.

diastólica $\geq 90 \mathrm{mmHg}$ ou uso de anti-hipertensivos. Pacientes diabéticos possuíam diagnóstico confirmado ou estavam em uso de medicação antidiabética no momento da inclusão no estudo.

\section{Protocolo de imageamento e análise dos dados}

Um cateter de ultrassom intracoronariano rotacional de $40 \mathrm{MHz}$ (Atlantis Pro, Boston Scientific, Boston, Estados Unidos) foi posicionado distal à lesão e recuado a $0,5 \mathrm{~mm} / \mathrm{s}$ até a referência proximal. Os contornos da membrana elástica externa (MEE), da placa e média (P\&M), e do lúmen foram gerados pela análise do corte tomográfico referente à ALM (Clearview, Boston Scientific, Boston, Estados Unidos), à área da P\&M (MEE$\mathrm{ALM})$ e à carga de placa $(\mathrm{P} \& \mathrm{M} / \mathrm{ALM})$ calculadas.

\section{Análise estatística}

A análise estatística foi realizada com auxílio do software StatView 5.0.1 (SAS Institute, Cary, Estados Unidos). Variáveis categóricas foram expressas por meio de números ou frequências e comparadas utilizando o teste de qui-quadrado. Variáveis contínuas foram reportadas na forma de média \pm um desvio padrão. $O$ teste $t$ de Student não-pareado foi utilizado para acessar diferenças entre dois ou mais conjuntos de dados com distribuição normal. A regressão linear simples foi utilizada para determinar a relação entre variáveis contínuas, e os valores de coeficiente de correlação (r) de Pearson foram determinados. Regressão multivariada em etapas foi utilizada para identificar preditores da área do ateroma, e coeficientes padronizados de regressão (Coef) foram calculados para quantificar a importância de cada variável independente no modelo final. Idade, parâmetros de perfil lipídico e fração de ejeção do ventrículo esquerdo foram incluídos no modelo como variáveis contínuas e as demais, como variáveis categóricas. Valores de probabilidade $<0,05$ foram considerados estatisticamente significantes.

\section{RESULTADOS}

\section{Características demográficas e lesionais}

As características demográficas e lesionais estão listadas na Tabela 1. O diâmetro de estenose das lesões analisadas foi de $57 \pm 9 \%$ (estimativa visual). Parâmetros de perfil lipídico estão listados na Tabela 2. No momento da inclusão no estudo, $83 \%$ dos pacientes estavam sob terapia com estatinas. 
TABELA 1

Características demográficas e lesionais (31 pacientes/35 lesões)

\begin{tabular}{|c|c|}
\hline \multicolumn{2}{|l|}{ Variáveis } \\
\hline Idade, anos (média \pm DP) & $64,9 \pm 10$ \\
\hline Sexo masculino, n (\%) & $27(75)$ \\
\hline Tabagistas, n (\%) & $7(19)$ \\
\hline Dislipidemias, n (\%) & $33(91)$ \\
\hline Hipertensão, n (\%) & $27(75)$ \\
\hline História familiar, n (\%) & $10(28)$ \\
\hline Diabetes, n (\%) & $8(22)$ \\
\hline IAM prévio, n (\%) & $7(19)$ \\
\hline Betabloqueadores, n (\%) & $20(56)$ \\
\hline Estatinas, $\mathrm{n}(\%)$ & $30(83)$ \\
\hline Inibidores da ECA, n (\%) & $21(58)$ \\
\hline Síndrome coronariana aguda, n (\%) & $7(19)$ \\
\hline \multicolumn{2}{|l|}{ Vaso-alvo, n (\%) } \\
\hline TCE & $3(8)$ \\
\hline DA & $21(60)$ \\
\hline $\mathrm{CX}$ & $6(17)$ \\
\hline CD & $5(14)$ \\
\hline \multicolumn{2}{|c|}{$\begin{array}{l}\mathrm{CD}=\text { artéria coronária direita; } \mathrm{CX}=\text { artéria circunflexa; } \mathrm{DA} \\
=\text { artéria descendente anterior; } \mathrm{DP}=\text { desvio padrão; } \mathrm{ECA}= \\
\text { enzima conversora de angiotensina; IAM = infarto agudo do } \\
\text { miocárdio; } \mathrm{n}=\text { número de pacientes; } \mathrm{TCE}=\text { tronco de } \\
\text { coronária esquerda. }\end{array}$} \\
\hline
\end{tabular}

TABELA 2

Valores médios dos parâmetros séricos de perfil lipídico e fração de ejeção do ventrículo esquerdo $(n=31)$

\begin{tabular}{lr}
\hline Perfil lipídico sérico & \\
\hline Colesterol total, mg/dl (média \pm DP) & $161,7 \pm 25$ \\
HDL-colesterol, mg/dl (média \pm DP) & $43,7 \pm 14$ \\
LDL-colesterol, mg/dl (média \pm DP) & $87,7 \pm 27$ \\
Triglicerídeos, mg/dl (média \pm DP) & $124,8 \pm 54$ \\
Fração de ejecão do & $66,8 \pm 8,3$ \\
ventrículo esquerdo, \% (média \pm DP) & \\
\hline
\end{tabular}

HDL-colesterol = colesterol de lipoproteína de alta densidade; LDL-colesterol = colesterol de lipoproteína de baixa densidade; $\mathrm{n}=$ número de pacientes.

\section{Análise com ultrassom intracoronariano}

Os dados da análise ao ultrassom intracoronariano estão listados na Tabela 3. A regressão simples univariada revelou associação inversa entre níveis de HDL e área da $P \& M(r=-0,40 ; P=0,02)$ e carga de placa $(r=-0,33 ; P=0,04)$ (Figura 1). Colesterol total, LDL ou triglicerídeos não apresentaram associação sig-
TABELA 3

Parâmetros de ultrassom intracoronariano $(\mathbf{n}=36)$

\begin{tabular}{lc}
\hline \multicolumn{2}{l}{ Parâmetros de USIC } \\
\hline $\begin{array}{l}\text { Diâmetro luminal mínimo, } \\
\text { mm (média } \pm \mathrm{DP})\end{array}$ & $2,26 \pm 0,41$ \\
Área do lúmen, $\mathrm{mm}^{2}$ (média $\left.\pm \mathrm{DP}\right)$ & $4,89 \pm 1,86$ \\
Área da MEE, $\mathrm{mm}^{2}$ (média $\left.\pm \mathrm{DP}\right)$ & $12,81 \pm 3,88$ \\
Área da P\&M, mm² (média $\pm \mathrm{DP})$ & $8,07 \pm 3,53$ \\
Carga de placa, \% (média $\pm \mathrm{DP})$ & $60,11 \pm 15,36$
\end{tabular}

MEE = membrana elástica externa; $\mathrm{n}=$ número de pacientes; P\&M = placa e média; USIC = ultrassom intracoronariano.

nificativa com nenhuma medida do ateroma. Os pacientes foram então separados de acordo com o corte "ótimo" dos parâmetros lipídicos e as medidas intraluminais foram comparadas. Pacientes com HDL $>50 \mathrm{mg} / \mathrm{dl}$ apresentaram menor área da P\&M $(4,9 \pm$ $3,5 \mathrm{~mm}^{2}$ vs. $\left.8,84 \pm 3,4 \mathrm{~mm}^{2} ; \mathrm{P}=0,03\right)$, e área da MEE $\left(9,4 \pm 3,2 \mathrm{~mm}^{2}\right.$ vs. $\left.13,9 \pm 3,7 \mathrm{~mm}^{2} ; \mathrm{P}=0,01\right)$ quando comparados a pacientes com HDL-colesterol $\leq 50 \mathrm{mg} / \mathrm{dl}$. Pacientes com LDL-colesterol $\geq 100$, colesterol total $\geq 200$ ou triglicerídeos $\geq 150$ apresentaram carga de placa e área da P\&M semelhantes quando comparados a pacientes apresentando níveis séricos abaixo desses valores. O mesmo foi observado quando os pacientes foram separados de acordo com o uso ou não de estatinas.

\section{Preditores independentes da carga de placa}

A regressão multivariada em etapas foi realizada para identificar preditores independentes da carga de placa, incluindo fatores de risco (idade, hipertensão, diabetes, dislipidemia, história familiar, tabagismo, síndrome coronariana aguda, infarto agudo do miocárdio prévio), parâmetros de perfil lipídico (HDL, LDL, triglicerídeos), uso de medicação anti-isquêmica (betabloqueadores, inibidores da enzima conversora de angiotensina, estatinas) e fração de ejeção do ventrículo esquerdo. O modelo final preditor da carga de placa foi composto por HDL (Coef $=-0,58 ; \mathrm{P}=0,001)$ e fração de ejeção do ventrículo esquerdo $($ Coef $=-0,40 ; P=0,02)$ (Tabela 4).

\section{DISCUSSÃO}

O primeiro estágio do processo aterosclerótico é a estria gordurosa, consistindo em sua maioria de macrófagos repletos de colesterol, derivado principalmente do LDL-colesterol. O segundo estágio consiste de placas fibrosas nas quais uma camada de tecido fibroso recobre um núcleo rico em lípides. A partir do momento em que a doença aterosclerótica está estabelecida, o papel das lipoproteínas ainda é pouco compreendido. Altos níveis séricos de LDL-colesterol 
Missel E, et al. Influência do Perfil Lipídico em Placas Ateroscleróticas Coronarianas Moderadas: Análise com Ultrassom Intracoronariano. Rev Bras Cardiol Invas. 2009;17(1):88-93.
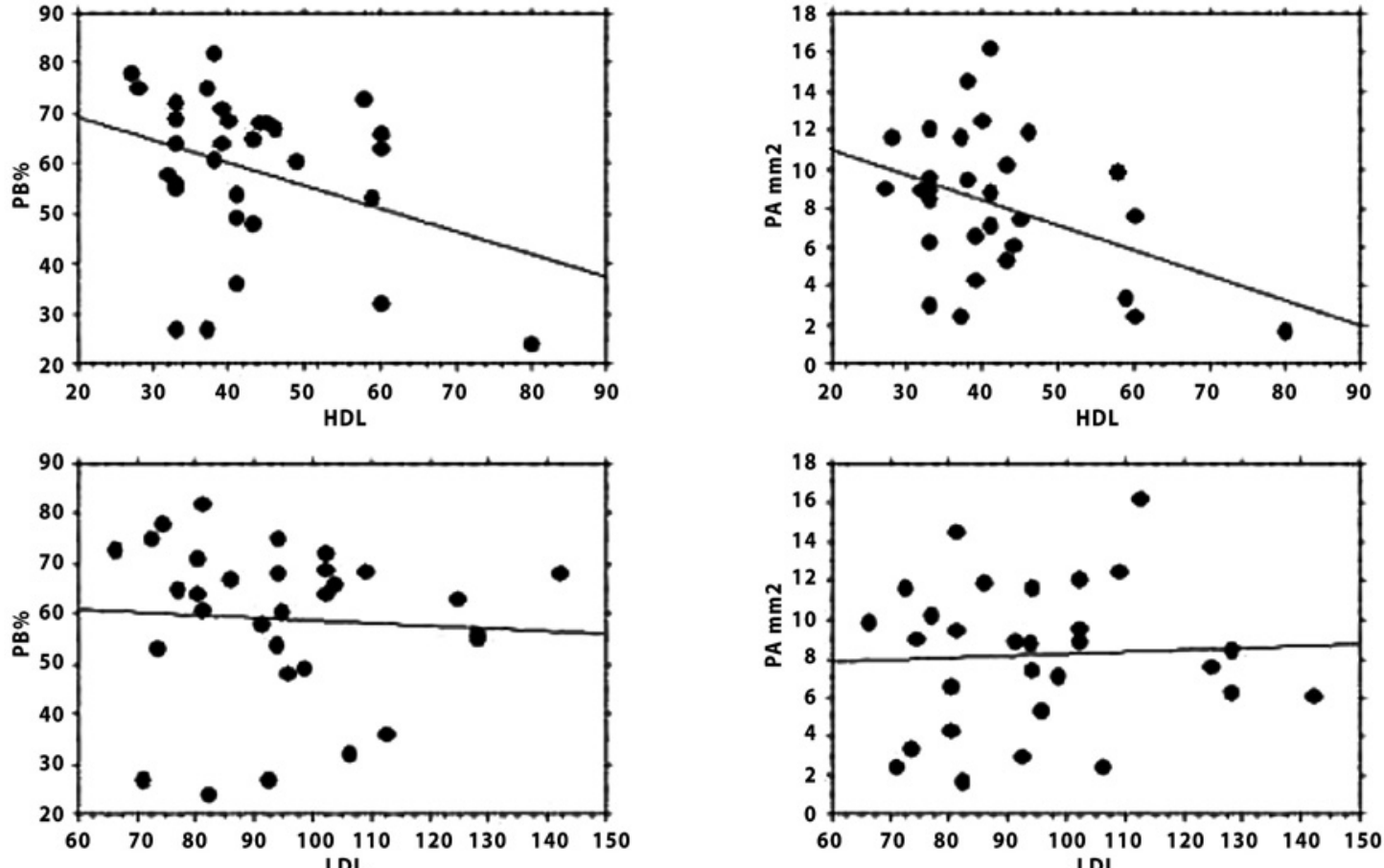

Figura 1 - Regressão simples mostrando associação significativa dos níveis séricos de colesterol de proteína de alta densidade (HDL-colesterol) com carga de placa $(P B)(r=-0,33 ; P=0,04)$ e área da $P \& M(P A)(r=-0,40 ; P=0,02)$. Em contrapartida, não houve correlação significativa dos níveis séricos de colesterol de proteína de baixa densidade (LDL-colesterol) com carga de placa $(\mathrm{PB})(r=-0,06 ; \mathrm{P}=0,73)$ e área da P\&M (PA) $(r=0,05 ; P=0,78)$

TABELA 4

Preditores independentes da carga de placa*

\begin{tabular}{lcccc} 
& Coeficiente padronizado & Coeficiente & Valor do $\boldsymbol{t}$ & P \\
\hline HDL-colesterol & $-0,58$ & $-0,63$ & 3,61 & 0,001 \\
Fração de ejeção do ventrículo esquerdo & $-0,40$ & $-0,64$ & 2,47 & 0,02 \\
\hline
\end{tabular}

*Regressão múltipla em etapas. Idade, índice de massa corporal, diabetes melito, hipertensão arterial sistêmica, dislipidemias, tabagismo, história familiar, síndrome coronariana aguda, LDL-colesterol, triglicerídeos, e uso de estatinas, betabloqueadores e inibidores da enzima conversora de angiotensina não atingiram correlação significativa para entrada no modelo.

HDL-colesterol = colesterol de lipoproteína de alta densidade; LDL-colesterol = colesterol de lipoproteína de baixa densidade.

aumentam a disponibilidade de LDL-colesterol na parede do vaso, permitindo aos macrófagos captarem avidamente colesterol das lipoproteínas associadas à apolipoproteína B, e ficarem presos no espaço subendotelial, aumentando o núcleo necrótico rico em lípides. Antagonicamente, o aumento dos níveis séricos de HDLcolesterol acelera o carreamento do excesso de colesterol das células periféricas para o fígado, onde serão removidos, potencialmente diminuindo o tamanho do núcleo lipídico por meio do transporte reverso de colesterol ${ }^{13}$.

A relação entre lípides séricos e extensão da doença aterosclerótica foi avaliada inicialmente por meio de angiografia. Em uma metanálise de estudos angiográficos acessando a efetividade de terapias hipolipemiantes distintas na regressão de placa, a terapia com estatinas objetivando diminuir os níveis de LDL-colesterol foi relacionada a menor incidência de eventos cardíacos quando comparada a outras terapias. Entretanto, essa modalidade de tratamento foi muito inferior a outras terapias avaliadas na habilidade de promover regressão vs. progressão da placa aterosclerótica (modificações do estilo de vida: odds ratio 10,6, P < 0,0001; estatinas: odds ratio $2,1, \mathrm{P}<0,0001$ vs. placebo $)^{14}$. Apesar de as modificações do estilo de vida serem bem conhecidas como medidas eficazes em aumentar os níveis séricos de HDL-colesterol, o efeito direto do 
Missel E, et al. Influência do Perfil Lipídico em Placas Ateroscleróticas Coronarianas Moderadas: Análise com Ultrassom Intracoronariano. Rev Bras Cardiol Invas. 2009;17(1):88-93.

HDL-colesterol na regressão/progressão de placa não foi avaliado diretamente neste estudo.

A relação do HDL-colesterol com a quantidade de placa aterosclerótica detectada pelo ultrassom intracoronariano foi descrita anteriormente em um estudo transversal de pacientes com hiperlipidemia familiar e aterosclerose "silenciosa" detectada pela angiografia ${ }^{15}$. Nessa análise, o HDL-colesterol foi o preditor mais robusto da carga de placa aterosclerótica no tronco da artéria coronária esquerda, na artéria descendente anterior e nas artérias ilíacas, em concordância com os achados de nosso estudo, que avaliou lesões angiograficamente moderadas na totalidade da árvore coronariana. De forma semelhante, em um estudo longitudinal acessando progressão/regressão de placa ao longo do tempo em 60 pacientes portadores de lesão em tronco de artéria coronária esquerda, os autores observaram relação inversa entre os níveis de HDLcolesterol em mudanças anuais no tamanho do ateroma $(r=-0,30 ; P=0,02)^{16}$. Em uma metanálise combinando os resultados para pacientes incluídos em quatro estudos prospectivos que utilizaram ultrassom intracoronariano para determinar mudanças no volume do ateroma durante o tratamento com estatinas, os autores observaram que a regressão de placa significativa só era alcançada se a diminuição dos níveis séricos de LDL-colesterol era acompanhada por aumento de 7,5\% dos níveis séricos de HDL-colesterol ${ }^{17}$. Os achados de nosso estudo transversal demonstrando que o $\mathrm{HDL}$ colesterol é o parâmetro lipídico determinante da carga de placa aterosclerótica, independentemente de outros parâmetros lipídicos, fatores de risco, medicações anti-isquêmicas ou estatinas, são consistentes com esse conceito. Adicionalmente, as terapias objetivando aumentar os níveis de HDL-colesterol têm demonstrado benefício adicional no combate à aterosclerose. No estudo randomizado controlado Arterial Biology for the Investigation of the Treatment Effects of Reducing Cholesterol (ARBITER) $2^{18}$, o ácido nicotínico foi associado à terapia com estatinas, demonstrando que a progressão da espessura intimal-medial de artérias carótidas foi retardada mais efetivamente se comparada ao emprego isolado de estatinas.

A fração de ejeção do ventrículo esquerdo, analisada como variável contínua, foi um dos preditores da carga de placa neste estudo, juntamente com os níveis séricos de HDL. Apesar de essa associação não ter sido descrita anteriormente, acreditamos que se deva ao fato de pacientes com função ventricular deteriorada em nossa população serem portadores de miocardiopatia isquêmica e, portanto, apresentarem padrão de doença aterosclerótica mais agressiva. Entretanto, o fato de pacientes com HDL baixo associado a função ventricular deteriorada apresentarem maior tamanho do ateroma deve ser mais bem esclarecido em estudos longitudinais posteriores, para se determinar se uma intervenção mais agressiva nos níveis de HDL pode acarretar benefício adicional nesse subgrupo de pacientes.

\section{Limitações do estudo}

O presente estudo possui várias limitações. A falta de uma avaliação seriada com ultrassom intracoronariano para acessar regressão/progressão de placa limita as conclusões deste estudo às de um estudo observacional, não-longitudinal, não podendo, portanto, inferir conclusões referentes à progressão/regressão de placa. Adicionalmente, não foi avaliada a ação de terapias objetivando aumentar os níveis de HDL, como uso de fibratos, ácido nicotínico ou mudanças no estilo de vida. Por último, como o estudo foi realizado com uma amostra pequena, estudos em maior escala são necessários para confirmar esses achados.

\section{CONCLUSÕES}

Nesta amostra de pacientes estudados, o HDL-colesterol é o parâmetro de perfil lipídico com maior impacto no tamanho do ateroma em lesões angiograficamente moderadas avaliadas ao ultrassom intracoronariano, independentemente dos níveis séricos de LDL-colesterol, do uso de estatinas ou de outros fatores de risco.

\section{CONFLITO DE INTERESSES}

Os autores declararam inexistência de conflito de interesses.

\section{REFERÊNCIAS BIBLIOGRÁFICAS}

1. The Lipid Research Clinics Coronary Primary Prevention Trial results. I. Reduction in incidence of coronary heart disease. JAMA. 1984;251(3):351-64.

2. The Lipid Research Clinics Coronary Primary Prevention Trial results. II. The relationship of reduction in incidence of coronary heart disease to cholesterol lowering. JAMA. 1984; 251(3):365-74.

3. Rossouw JE, Lewis B, Rifkind BM. The value of lowering cholesterol after myocardial infarction. N Engl J Med. 1990; 323(16):1112-9.

4. Pekkanen J, Linn S, Heiss G, Suchindran CM, Leon A, Rifkind BM, et al. Ten-year mortality from cardiovascular disease in relation to cholesterol level among men with and without preexisting cardiovascular disease. N Engl J Med. 1990;322(24):1700-7.

5. Wong ND, Wilson PW, Kannel WB. Serum cholesterol as a prognostic factor after myocardial infarction: the Framingham Study. Ann Intern Med. 1991;115(9):687-93.

6. Wilson PW, D'Agostino RB, Levy D, Belanger AM, Silbershatz $\mathrm{H}$, Kannel WB. Prediction of coronary heart disease using risk factor categories. Circulation. 1998;97(18):1837-47.

7. Blankenhorn DH, Azen SP, Kramsch DM, Mack WJ, CashinHemphill L, Hodis HN, et al.; MARS Research Group. Coronary angiographic changes with lovastatin therapy. The Monitored Atherosclerosis Regression Study (MARS). Ann Intern Med. 1993;119(10):969-76.

8. Waters D, Higginson L, Gladstone P, Kimball B, Le May M, Boccuzzi SJ, et al. Effects of monotherapy with HMG-CoA reductase inhibitor on the progression of coronary atherosclerosis as assessed by serial quantitative arteriography. The Canadian Coronary Atherosclerosis Intervention Trial. Circulation. 1994;89(3):959-68. 
Missel E, et al. Influência do Perfil Lipídico em Placas Ateroscleróticas Coronarianas Moderadas: Análise com Ultrassom Intracoronariano. Rev Bras Cardiol Invas. 2009;17(1):88-93.

9. Jukema JW, Bruschke AV, van Boven AJ, Reiber JH, Bal $\mathrm{ET}$, Zwinderman $\mathrm{AH}$, et al. Effects of lipid lowering by pravastatin on progression and regression of coronary artery disease in symptomatic men with normal to moderately elevated serum cholesterol levels. The Regression Growth Evaluation Statin Study (REGRESS). Circulation. 1995; 91(10):2528-40.

10. Pitt B, Mancini GB, Ellis SG, Rosman HS, Park JS, McGovern ME. Pravastatin limitation of atherosclerosis in the coronary arteries (PLAC I): reduction in atherosclerosis progression and clinical events. PLAC I investigation. J Am Coll Cardiol. 1995;26(5):1133-9.

11. Brown G, Albers JJ, Fisher LD, Schaefer SM, Lin JT, Kaplan C, et al. Regression of coronary artery disease as a result of intensive lipid-lowering therapy in men with high levels of apolipoprotein B. N Engl J Med. 1990; 323(19):1289-98.

12. Effect of simvastatin on coronary atheroma: the Multicentre Anti-Atheroma Study (MAAS). Lancet. 1994;344(8923):633-8.

13. Forrester JS. Prevention of plaque rupture: a new paradigm of therapy. Ann Intern Med. 2002;137(10):823-33.

14. Holmes CL, Schulzer M, Mancini GB. Angiographic results of lipid-lowering trials: a systematic review and meta-analysis. In: Grundy SM, editor. Cholesterol-lowering therapy: evaluation of clinical trial evidence. New York: Marcel Dekker Inc.; 2000. p.191-220.

15. Hausmann D, Johnson JA, Sudhir K, Mullen WL, Friedrich G, Fitzgerald PJ, et al. Angiographically silent atherosclerosis detected by intravascular ultrasound in patients with familial hypercholesterolemia and familial combined hyperlipidemia: correlation with high density lipoproteins. J Am Coll Cardiol. 1996;27(7):1562-70.

16. von Birgelen C, Hartmann M, Mintz GS, Baumgart D, Schmermund A, Erbel R. Relation between progression and regression of atherosclerotic left main coronary artery disease and serum cholesterol levels as assessed with serial long-term ( $\geq 12$ months) follow-up intravascular ultrasound. Circulation. 2003;108(22):2757-62.

17. Nicholls SJ, Tuzcu EM, Sipahi I, Grasso AW, Schoenhagen $\mathrm{P}$, Hu T, et al. Statins, high-density lipoprotein cholesterol, and regression of coronary atherosclerosis. JAMA. 2007; 297(5):499-508.

18. Taylor AJ, Sullenberger LE, Lee HJ, Lee JK, Grace KA. Arterial Biology for the Investigation of the Treatment Effects of Reducing Cholesterol (ARBITER) 2: a double-blind, placebocontrolled study of extended-release niacin on atherosclerosis progression in secondary prevention patients treated with statins. Circulation. 2004;110(23):3512-7. 